\title{
AC Magnetic Susceptibility Study of Fe and Co Based Nanoparticles
}

\author{
A. Zeleñákováa ${ }^{a, *}$, J. KováČ ${ }^{b}, \mathrm{~V}$. ZeleñáK ${ }^{c}$ \\ ${ }^{a}$ Institute of Physics, Faculty of Sciences, P.J. Šafárik University, Park Angelinum 9, 04154 Košice, Slovakia \\ ${ }^{b}$ Institute of Experimental Physics, Slovak Academy of Sciences, Watsonova 41, 04154 Košice, Slovakia \\ ${ }^{c}$ Institute of Chemistry, Faculty of Sciences, P.J. Šafárik University, Moyzesova 11, 04154 Košice, Slovakia
}

\begin{abstract}
We have investigated magnetic properties of three different samples composed of magnetic Fe or Co core and protective $\mathrm{Au}$ or Pt metallic shell, namely Fe@Au, Co@Au and Fe@Pt samples, with sizes of about 10 nm, 8 nm and $5 \mathrm{~nm}$ respectively. We have measured and analysed the experimental data of real and imaginary component of ac susceptibility through theoretical laws for non-interacting and interacting particles and we have estimated the strength of their magnetic interactions. Our analyses showed the increase of inter-particle interactions with decreasing particles size.
\end{abstract}

DOI: 10.12693/APhysPolA.126.222

PACS: 75.75.Fk, 81.05.Rm, 75.40.Cx

\section{Introduction}

Mono-domain metal nanoparticles based on iron and cobalt are of great interest due to their intrinsic physical properties, such as macroscopic quantum tunnelling (MQT), quantum size effects, giant magnetic moment and surface spin frustration interplay between the surface and core atoms. If magnetic interactions between the particles are not negligible, they can have a significant influence on the superparamagnetic relaxation [1]. Furthermore, the spin structure of nanoparticles can be affected by inter-particle interactions. An assembly of nanoparticles coupled by sufficiently weak inter-particle interactions show a superparamagnetic behaviour, while stronger interactions between densely packed nanoparticles can stabilize superspin glass or superferromagnetic state [2]. One of the powerful ways to get a better insight into the nature of inter-particle interactions and to estimate their strength, is the analysis of ac magnetic susceptibility [3].

In our work, we have investigated the strength of dipolar magnetic interactions between nanoparticles in three different samples using the ac complex susceptibility measurements and their analysis through theoretical laws.

\section{Experimental}

Nanoparticle samples were prepared from water solutions of $\mathrm{HAuCl}_{4}, \mathrm{H}_{2} \mathrm{PtCl}_{6}, \mathrm{Co}\left(\mathrm{NO}_{3}\right)_{2}, \mathrm{Fe}\left(\mathrm{NO}_{3}\right)_{2}$ using the reverse micelle concept. CTAB was used as the surfactant and octane as the oil phase in the formation of reverse micelles. Reduction of metal salts was done by $\mathrm{NaBH}_{4}$. Three different nanoparticle systems were prepared and studied: Fe nanoparticles coated by $\mathrm{Au}$ (Fe@Au, sample 1), Co nanoparticles coated by $\mathrm{Au}$ (Co@Au, sample 2) and Fe nanoparticles coated by Pt (Fe@Pt, sample 3 ).

*corresponding author; e-mail: adriana.zelenakova@upjs.sk
The structural studies were realised by the $\mathrm{X}$ ray diffraction (XRD), X-ray Near Edge Spectroscopy (XANES), and by High-resolution transmission electron microscopy (HRTEM).

Magnetic properties were investigated using a SQUID (Superconducting Quantum Interference Device) apparatus in the external dc field up to $5 \mathrm{~T}$ and in the temperature range of $2-300 \mathrm{~K}[4]$. The complex ac magnetic susceptibility $\chi^{\prime}(T)-i \chi^{\prime \prime}(T)$, where $\chi^{\prime}$ represents in-phase ac susceptibility (real part) and $\chi^{\prime \prime}$ out-of-phase (imaginary part) susceptibility, was registered by the same instruments in the temperature interval $2-300 \mathrm{~K}$ and in the frequency interval $0.1-1000 \mathrm{~Hz}$.

\section{Results and discussion}

The structural characterization using XRD and XANES measurements confirmed the composition of the prepared samples and their nanocrystalline character. The HRTEM measurements showed the spherical shape of the particles with the size about $10 \mathrm{~nm}$ for Fe@Au sample, $8 \mathrm{~nm}$ for $\mathrm{Co} @ A u$ sample and $5 \mathrm{~nm}$ for Fe@Pt sample.

The measurements of DC magnetic susceptibility in ZFC (zero field cooling) and FC (field cooling) regimes confirmed the superparamagnetic character in all three samples and the existence of blocking temperature $T_{B}$, below which the magnetic moments of the particles are blocked along the external field direction. Since the presence of relaxation process was observed in all studied nanoparticle samples, we have focused on the measurements and analysis of ac complex magnetic susceptibility, to better understand the dynamic properties of magnetic materials.

Figure 1 shows the data of ac susceptibility measured for the sample $1(\mathrm{Fe} @ \mathrm{Au})$ at frequencies in the range 1$1000 \mathrm{~Hz}$. As can be seen, the data exhibits the behaviour typical for blocking/freezing processes, such as the presence of a maximum in in-phase ac susceptibility $\chi^{\prime}(T)$ (real part) (linked to the left vertical axis), which shifts towards higher temperatures with increasing frequency, 


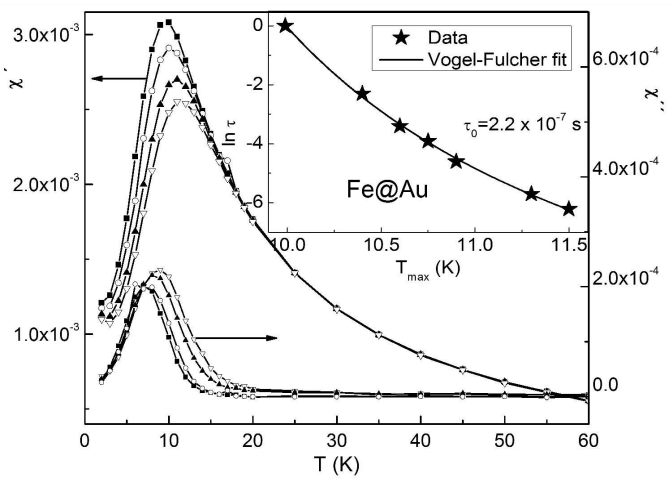

Fig. 1. Temperature dependences of in-phase $\chi^{\prime}(T)$ (linked left) and out-of-phase $\chi^{\prime \prime}(T)$ (linked right) ac susceptibility of sample Fe@Au, measured at different frequencies from the range $1 \mathrm{~Hz}$ (black squares) to $1000 \mathrm{~Hz}$ (white triangles). Inset shows Vogel-Fulcher fit of experimental data.

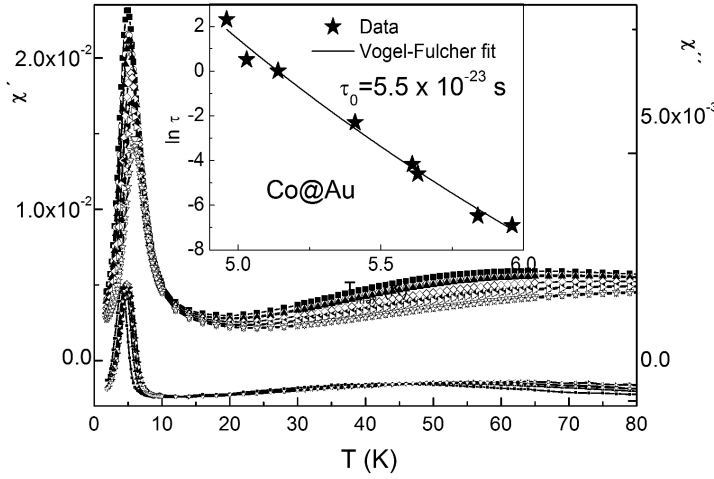

Fig. 2. Temperature dependences of $\chi^{\prime}(T)$ and $\chi^{\prime \prime}(T)$ of the sample Co@Au, measured at different frequencies from the range $1 \mathrm{~Hz}$ (black squares) to $1000 \mathrm{~Hz}$ (white triangles). Inset shows Vogel-Fulcher fit of experimental data.

and the out-of phase ac susceptibility $\chi^{\prime \prime}(T)$ (imaginary part), which exhibits a sudden onset near $T_{\max }$.

Figure 2 shows the data of ac complex susceptibility of sample $2(\mathrm{Co} @ \mathrm{Au})$, for which the size of particles was smaller in comparison with sample 1 . The existence of two maxima can be clearly seen in Fig. 2, a narrow and sharp maximum centred at $7 \mathrm{~K}$ and second, broader one centred at $65 \mathrm{~K}$. The ac susceptibility measurements of the sample 3 (Fe@Pt) also exhibited a frequency dependent maximum (see Fig. 3), which shifted to the higher temperatures with increasing frequency, similarly to samples 1 and 2 . The shape of temperature dependencies of ac susceptibility suggests, that at temperatures below $2 \mathrm{~K}$ an additional maximum could exist, similar to that observed for sample 2. We have analysed the existing maxima in ac susceptibility measurements from all three samples using three theories, (i) the Neel-Arrhenius law [2], describing the non-interacting nanoparticles, (ii) the Vogel-Fulcher law [2], confirming the interacting nanoparticle system with dipolar magnetic interaction and (iii) the critical power law [2], show- ing on the presence of collective behaviour in nanoparticle systems. Such analyses showed that in the sample 1 the weak interactions were present, whereas in the sample 3 the strong dipolar magnetic interactions existed. The analysis of the sharp, narrow maximum in the sample 2, using the dynamic scaling hypothesis, showed the existence of conventional critical slowing down mechanism, which suggests the existence of superspin glass phase in sample 2 .

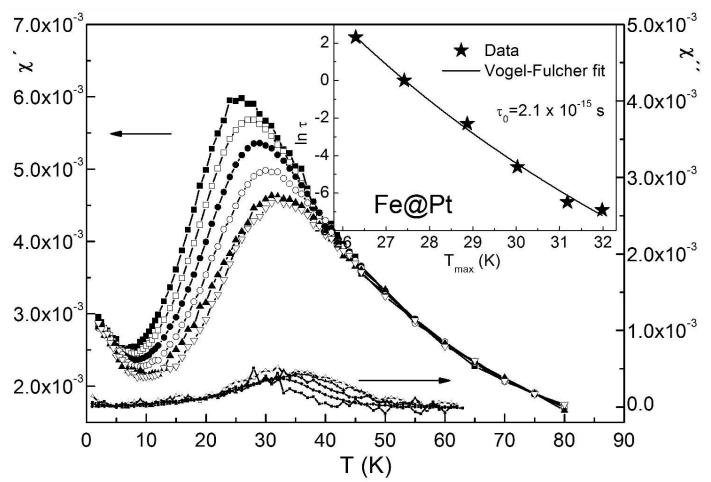

Fig. 3. Temperature dependences of in-phase $\chi^{\prime}(T)$ (linked left) and out-of-phase $\chi^{\prime \prime}(T)$ (linked right) ac susceptibility in the sample Fe@Pt, measured at different frequencies from the range $1 \mathrm{~Hz}$ (black squares) to $1000 \mathrm{~Hz}$ (white triangles). Inset shows Vogel-Fulcher fit of experimental data.

\section{Conclusions}

We have prepared three nanoparticle samples Fe@Au, $\mathrm{Co} @ \mathrm{Au}, \mathrm{Fe} @ \mathrm{Pt}$ and studied their dynamic properties. The analyses of experimental data of ac complex susceptibility using the theoretical models showed the existence of the weak magnetic interactions in the Fe@Au sample, the existence of the superspin glass phase in Co@Au sample and the presence of strong dipolar interactions in the sample Fe@Pt. The observed increasing strength of the interparticle interactions from sample Fe@Au to sample Fe@Pt correlates with the decreasing particle size in these samples.

\section{Acknowledgments}

This work was supported by the VEGA projects No. $1 / 0583 / 11$ and No. $1 / 0861 / 12$, Slovak Research and Development Agency under the contracts APVV-0222-10 and APVV-0132-11 and by the ERDF EU grant under Contract No. ITMS 262201200005. The authors (A.Z and V.Z) would like to thank DESY/HASYLAB project under No. I-20110282 EC.

\section{References}

[1] S. Morup, M.F. Hansen, C. Frandsen, Beilstein J. Nanotechnol 1, 182 (2010).

[2] S. Bedanta, W. Kleemann, J. Phys. D: Appl. Phys 42, 013001 (2009).

[3] Y. Bitla, S.N. Kaul, L. Fernández Barquín, Phys. Rev. B 86, 094405 (2012).

[4] A. Zelenakova, J. Kovac, V. Zelenak, Acta Phys. Pol. A 115, 357 (2009). 